\title{
ANALYSIS OF CHAOTIC WANDERING OF PHASE PATTERNS IN A TWO-DIMENSIONAL COUPLED CHAOTIC CIRCUITS NETWORK
}

\author{
Yoshifumi NISHIO and Akio USHIDA \\ Dept. of Electrical and Electronic Engineering, Tokushima University \\ 2-1 Minami-Josanjima, Tokushima, 770-8506 Japan \\ Email: nishio@ee.tokushima-u.ac.jp
}

\section{INTRODUCTION}

Studies on spatio-temporal phenomena observed from coupled chaotic nctworks, namely coupled systems of many chaotic cells, are classified into two categories; namely discrete time systems and continuous time systems. For discrete time systems Kaneko's coupled map lattice is the most interesting and well-studied system [1]. He discovered various nonlinear spatiotemporal chaotic phenomena such as clustering, Brownian motion of defect and so on. Also Aihara's chaos neural network is the most important chaotic network from an engineering point of view [2]. His study indicated new possibility of engineering applications of chaotic networks, namely dynamical search of patterns embedded in neural networks utilizing chaotic wandering. Further, application of chaos neural network to optimization problems is widely studied. However, for continuous time systems there have been few studies on spatial patterns observed after vanishing the effect of initial patterns. Thercforc, in order to fill the gap between the studies of discrete-time mathematical abstract and studies of continuous-time real physical systems, it is important to investigate simple continuoustime coupled chaotic circuits networks generating clustering or pattern switching phenomenon.

We have proposed a continuous-time coupled chaotic circuits network [3]-[6] and have reported generating spatial patterns, irregular self-switching phenomenon of spatial patterns, pattern control and so on. The important feature of our chaotic network is its coupling_structure. Namely adjacent four chaotic circuits are coupled by one resistor. Because such a coupling exhibited quasi-synchronization with phase difference [7], various spatial patterns could be generated in the chaotic network. Therefore, the network would be a good model to make clear physical mechanism of spatio-temporal chaotic phenomena in continuoustime systems. However, chaotic wandering phenomena of phase patterns observed from a relatively larger size of the network has not been investigated.

In this study, we investigate chaotic wandering phenomena of spatial patterns characterized by phase differences in the coupled chaotic circuits network with $10 \times 10$ size. Snapshots of the Poincaré map tell how switchings of phase states start and spread over the network. Further, we can show that an independent angular variables derived from higher-dimensional Poincaré map can be effectively utilized in order to check the switching of phase states around coupling resistors.

\section{CHAOTIC CIRCUITS NETWORK}

Fig. 1 shows coupled chaotic circuits network. We approximate the $i-v$ characteristics of the diodes by a two-segment piccewise-linear function as

$$
v_{d}\left(i_{m, n}\right)=0.5\left(r_{d} i_{m, n}+E-\left|r_{d} i_{m, n}-E\right|\right) .
$$

By changing the variables and parameters,

$$
\begin{gathered}
j_{m, n}^{a}=\sqrt{\frac{C}{L_{1}}} E w_{m, n}^{a}, \quad j_{m, n}^{b}=\sqrt{\frac{C}{L_{1}}} E w_{m, n}^{b}, \\
j_{m, n}^{c}=\sqrt{\frac{C}{L_{1}}} E w_{m, n}^{c}, \quad j_{m, n}^{d}=\sqrt{\frac{C}{L_{1}}} E w_{m, n}^{d}, \\
i_{m, n}=\sqrt{\frac{C}{L_{1}}} E y_{m, n}, \quad v_{m, n}=E z_{m, n}, \\
t=\sqrt{L_{1} C} \tau, \quad \alpha=\frac{L_{1}}{L_{2}}, \quad \beta=r \sqrt{\frac{C}{L_{1}}}, \\
\gamma=R \sqrt{\frac{C}{L_{1}}}, \quad \delta=r_{d} \sqrt{\frac{C}{L_{1}}}
\end{gathered}
$$

the normalized circuit equations of the network with $N_{1} \times N_{2}$ size are described as

$$
\begin{aligned}
x_{m, n}= & w_{m, n}^{a}+w_{m, n}^{b}+w_{m, n}^{c}+w_{m, n}^{d} \\
\frac{d y_{m, n}}{d \tau}= & \alpha\left\{\beta\left(x_{m, n}+y_{m, n}\right)-z_{m, n}-f\left(y_{m, n}\right)\right\} \\
\frac{d z_{m, n}}{d \tau}= & x_{m, n}+y_{m, n} \\
\frac{d w_{m, n}^{a}}{d \tau}= & \beta\left(x_{m, n}+y_{m, n}\right)-z_{m, n}-\gamma\left(w_{m, n}^{a}\right. \\
& \left.+w_{m, n-1}^{b}+w_{m-1, n}^{c}+w_{m-1, n-1}^{d}\right) \\
\frac{d w_{m, n}^{b}}{d \tau}= & \beta\left(x_{m, n}+y_{m, n}\right)-z_{m, n}-\gamma\left(w_{m, n+1}^{a}\right. \\
& \left.+w_{m, n}^{b}+w_{m-1, n+1}^{c}+w_{m-1, n}^{d}\right)
\end{aligned}
$$




$$
\begin{aligned}
\frac{d w_{m, n}^{c}}{d \tau}= & \beta\left(x_{m, n}+y_{m, n}\right)-z_{m, n}-\gamma\left(w_{m+1, n}^{a}\right. \\
& \left.+w_{m+1, n-1}^{b}+w_{m, n}^{c}+w_{m, n-1}^{d}\right) \\
\frac{d w_{m, n}^{d}}{d \tau}= & \beta\left(x_{m, n}+y_{m, n}\right)-z_{m, n}-\gamma\left(w_{m+1, n+1}^{a}\right. \\
& \left.+w_{m+1, n}^{b}+w_{m, n+1}^{c}+w_{m, n}^{d}\right) \\
\frac{d w_{m, n}^{d}}{d \tau}= & \beta\left(x_{m, n}+y_{m, n}\right)-z_{m, n}-\gamma\left(w_{m+1, n+1}^{a}\right. \\
& \left.+w_{m+1, n}^{b}+w_{m, n+1}^{c}+w_{m, n}^{d}\right)
\end{aligned}
$$

$$
\left(m=1,2,3 \cdots N_{1} \text { and } n=1,2,3, \cdots N_{2}\right)
$$

where

$$
f\left(y_{m, n}\right)=\frac{\delta y_{m, n}+1-\left|\delta y_{m, n}-1\right|}{2},
$$

$w_{0, n}^{c}=w_{0, n}^{d}=w_{m, 0}^{b}=w_{m, 0}^{d}=w_{N_{1}+1, n}^{a}=w_{N_{1}+1, n}^{b}=$ $w_{m, N_{2}+1}^{a}=w_{m, N_{2}+1}^{c}=0$ and the value of $\gamma$ must be neglected for the calculation of the inductors on the edge.

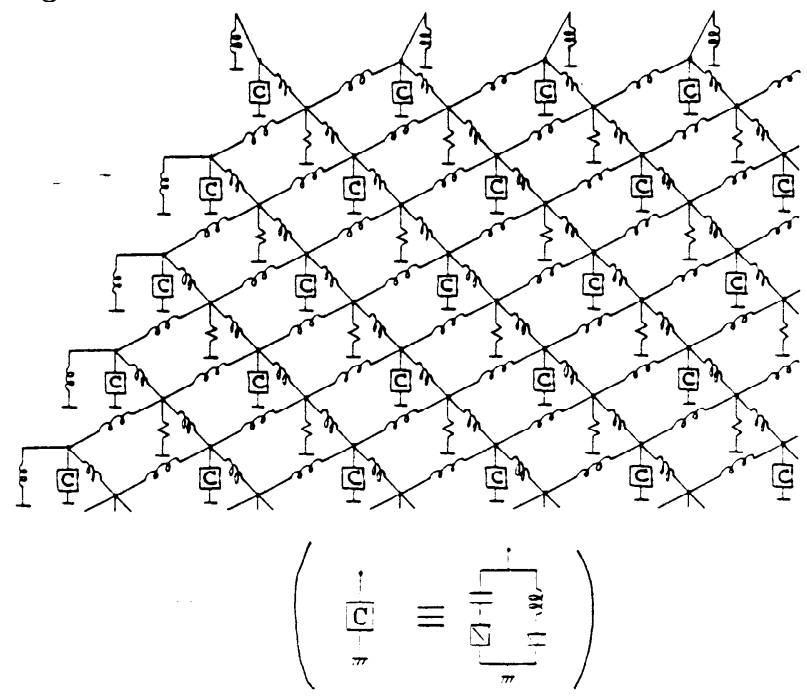

(a)

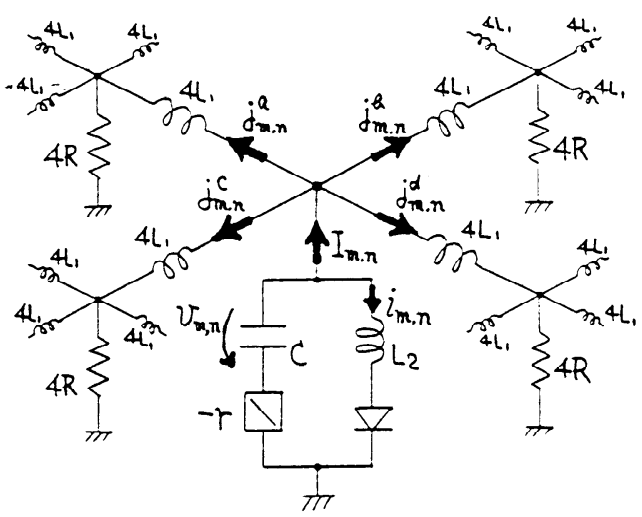

(b)

Fig. 1 (a) Chaotic circuits network. (b) Magnification around a cell at position $(m, n)$.
All of the results in this study are obtained by calculating Eq. (3) using the Runge-Kutta method with step size $h=0.005$. In the following computer calculations we fix the parameters $\alpha=7.0, \beta=0.13$ and $\delta=50.0$ and vary the coupling parameter $\gamma$ as a control parameter.

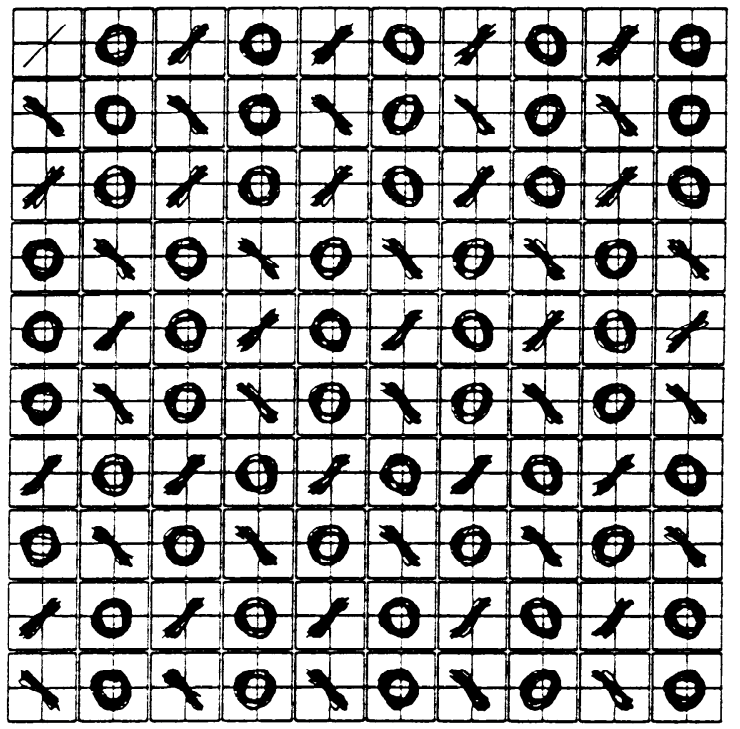

Fig. 2 An example of phase patterns observed from the chaotic circuits network with $10 \times 10$ size $(\gamma=0.30)$.

\section{SPATIAL PATTERNS}

Fig. 2 shows an example of frozen spatial patterns observed from the network with $10 \times 10$ size. In Fig. 2 all horizontal axes are $x_{1,1}$ and vertical axis of the attractor located $(m, n)$ is $x_{m, n}$. Hence, if the attractor located $(m, n)$ is almost on $45^{\circ}$ line, $x_{m, n}$ is almost synchronized to $x_{1,1}$. While, if the attractor located $(m, n)$ is almost on $-45^{\circ}$ line, $x_{m, n}$ is almost synchronized to $x_{1,1}$ with $\pi$ phase difference. If the attractor is almost on a circle, the phase sift is $\pm 90^{\circ}$. In Fig. 2 we can see that four phase synchronizations around coupling resistors can generate spatial patterns. Under the assumption that four-phase synchronizations are generated around all coupling resistors, we can prove that the number of phase patterns appearing in $N_{1} \times N_{2}$ network is $6\left(2^{N_{1}-2}+2^{N_{2}-2}-1\right)$. This means that $10 \times 10$ network can generate 3,066 different frozen spatial patterns and that $20 \times 20$ network generates $3,145,722$ patterns.

\section{CHAOTIC WANDERING}

Further, by tuning coupling parameter, we can observe chaotic wandering phenomenon of phase patterns in the network. In order to investigate how switchings of phase states start and spread over the network, we define the Poincaré section as $z_{1,1}=0$ and $x_{1,1}<0$. 
Fig. 3 shows the projections of the Poincaré map onto $\left(x_{m, n}-z_{m, n}\right)$ planes, which corresponds to the result in Fig. 2. Because the chaotic attractor observed from each subcircuit is strongly constrained onto the $(x-z)$ plane when the diode is off, this figure almost corresponds to the phase differences between the subcircuit located $(1,1)$ and the others.

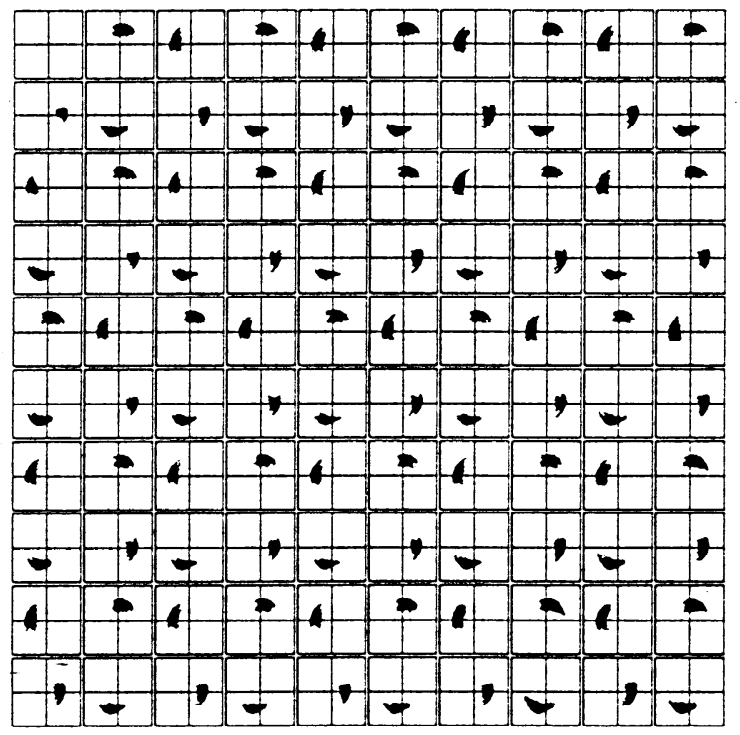

Fig. 3 Poincaré map of the phase pattern in Fig. 2 $(\gamma=0.30)$.

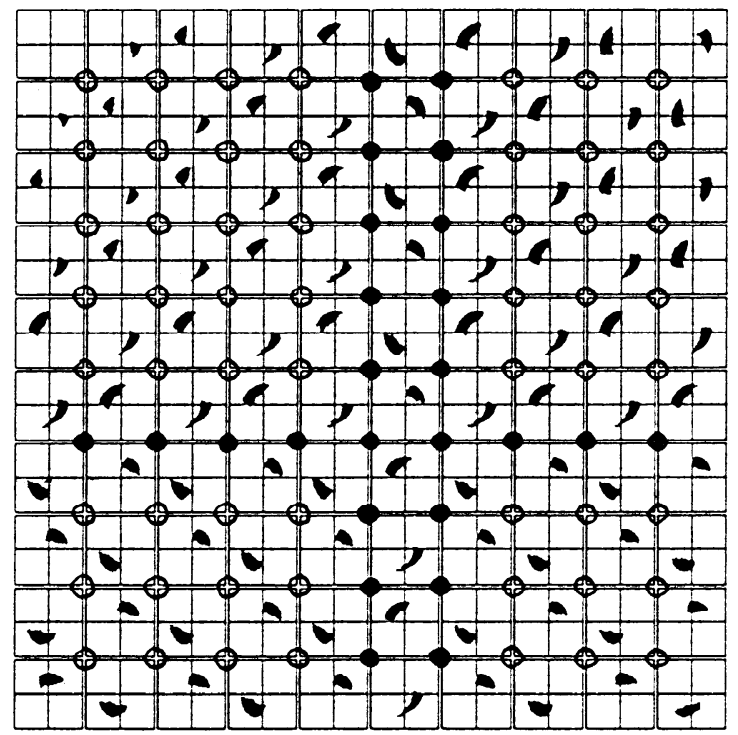

Fig. 4 Phase Pattern including in- and oppositephases synchronizations and four-phase synchronizations $(\gamma=0.10)$.

By computer simulations we found two critical coupling parameter values $\gamma_{C 1}$ and $\gamma_{C 2}$ at which observed phenomena change qualitatively. Namely, for $\gamma<\gamma_{C 1} \simeq 0.15$, in- and opposite-phases synchronizations [3] as well as four-phase synchronizations are in- cluded in frozen patterns. Fig. 4 shows an example of such frozen patterns. Four-phase synchronizations are observed around coupling resistors indicated as • , while in- and opposite-phases synchronizations are observed around coupling resistors indicated as o. For

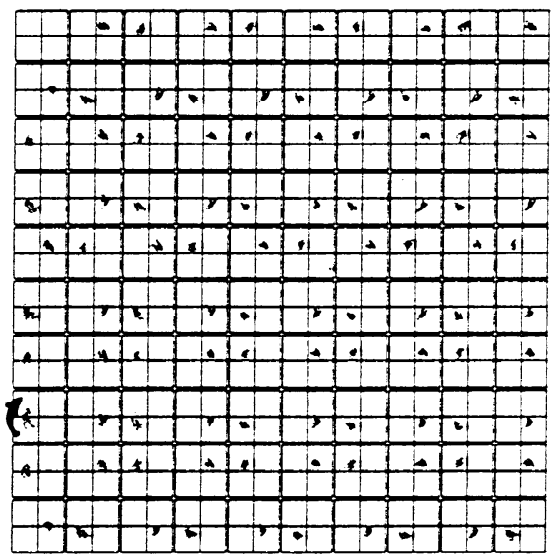

(a)

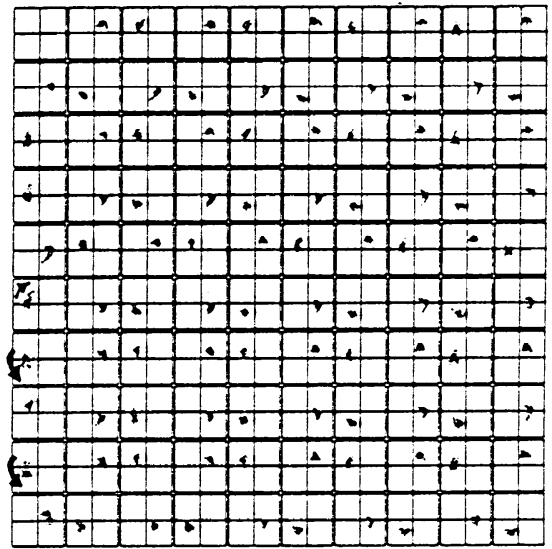

(b)

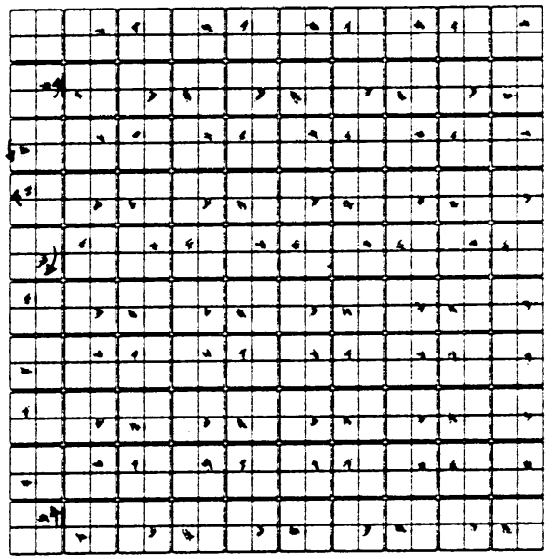

(c)

Fig. 5 Chaotic wandering of phase patterns. (a) $n=6,300 \sim 6,400$. (b) $n=6,400 \sim$ 6,500. (c) $n=6,500 \sim 6,600$. 
this parameter range spatial patterns including only four-phase synchronizations as Fig. 3 are also stable. Hence, if we give random initial conditions for the network, phase states settle down to one of a number of stable frozen patterns. For $\gamma_{C 1}<\gamma<\gamma_{C 2} \simeq 0.38$, inand opposite-phases synchronizations become unstable. If we give initial conditions corresponding to Fig. 4, the initial spatial pattern changes and will settles down to one of $6\left(2^{N_{1}-2}+2^{N_{2}-2}-1\right)$ spatial patterns after unpredicted chaotic wandering. For $\gamma_{C 2}<\gamma$, four-phase synchronizations also become unstable and no stable spatial patterns exist. Different spatial patterns appear but they disappear after a while. For relatively larger value of $\gamma$, well-organized frozen patterns are rarcly observed. In this case we can always find some partlydisturbed areas in the network. Though the area may be well-organized after a while, another area will starts to stir. Snapshots of the Poincaré map can show how switchings of phase states start and spread over the network. Fig. 5 shows a switching originated at $(1,8)$ generates turbulence along the edge of the network.

Finally, in order to get information of switchings of local phase patterns, we define the following independent variables from the discrete data of $x_{m, n}$ and $z_{m, n}$ on the Poincaré map.

$$
\varphi_{m, n}(n)=\left\{\begin{array}{l}
\pi-\tan ^{-1} \frac{z_{m, n}(n)}{x_{m, n}(n)} \\
\cdots+x_{m, n}(n)>0 \\
\pi+\tan ^{-1} \frac{z_{m, n}(n)}{x_{m, n}(n)} x_{m, n}(n)<0 \\
\cdots \quad x_{m}
\end{array}\right.
$$

By using these dependent angular variables, we can count the number of switching generated around all coupling resistors. In Fig. 6 the depth of gray at a position denotes switching number during 10,000 iterations of the Poincare map around the coupling resistor at the position. These data are utilized for statistical analysis of the chaotic wandering phenomenon.

\section{CONCLUSIONS}

In this study, we have investigated chaotic wandering phenomena of spatial patterns characterized by phase differences in a coupled chaotic circuits network. We showed that independent angular variables derived from higher-dimensional Poincaré map could be effectively utilized to check the switchings of phase states.

\section{ACKNOWLEDGMENT}

This work was partly supported by the Research Grants for Incentive Research from the Yazaki Memorial Foundation for Science and Technology.

\section{REFERENCES}

[1] K. Kaneko (ed.), Theory and Applications of Coupled Map Lattices, John Wiley \& Sons, Chichester, 1993.

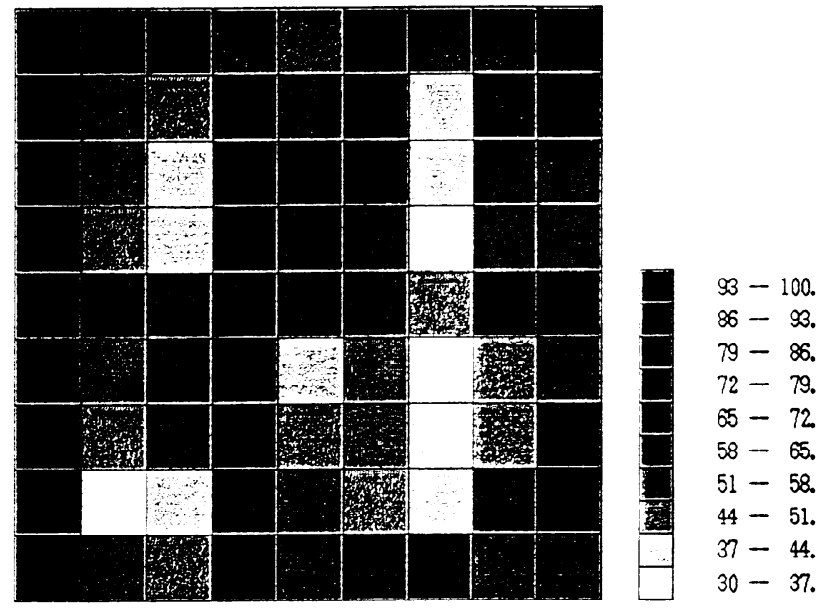

(a)

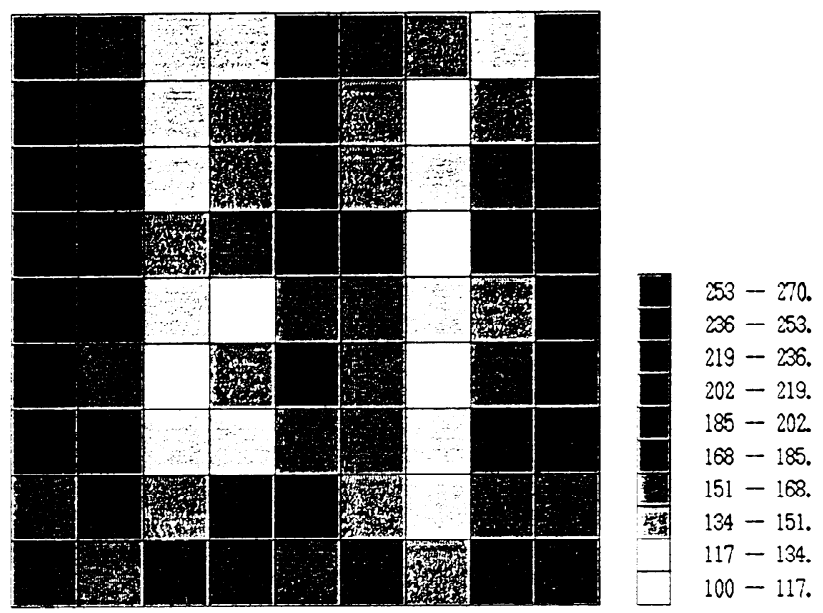

(b)

Fig. 6 Switching number in coupled chaotic network. (a) $\gamma=0.39$. (b) $\gamma=0.40$.

[2] K. Aihara et al., "Chaotic Neural Networks," Phys. Lett. A, vol. 144, no. 6\&7, pp. 333-340, 1990.

[3] Y. Nishio et al., "Spatio-Temporal Chaos in Simple Coupled Chaotic Circuits," IEEE Trans. CAS-I, vol. 42 , no. 10 , pp. 678-686, 1995.

[4] Y. Nishio et al., "On Synchronization Phenomena in Coupled Chaotic Circuits Networks," Proc. ISCAS'96, vol. 3, pp. 92-95, 1996.

[5] Y. Nishio et al., "Pattern Control in a Coupled Chaotic Network and its Possible Application in Communications," Proc. ISCAS'97, vol. 2, pp. 1037-1040, 1997.

[6] Y. Nishio et al., "On Phase Pattern in a Two-Dimensional Coupled Chaotic Network," Proc. NOLTA'98, vol. 1, pp. 31-34, 1998.

[7] Y. Nishio et al., "Quasi-Synchronization Phenomena in Chaotic Circuits Coupled by One Resistor," IEEE Trans. CAS-I, vol. 43, no. 6, pp. 491-496, 1996. 